\title{
Mechanical Ventilator Using Motorized Bellow
}

\author{
H. Hirani ${ }^{1}$ \\ Received: 15 April 2020 / Revised: 28 May 2020 / Accepted: 29 May 2020 / Published online: 11 June 2020 \\ (C) Indian National Academy of Engineering 2020
}

\begin{abstract}
The present work describes the state of art in India with respect to the development of low-cost mechanical ventilators. It highlights the bare minimum specifications that are required in a ventilator to fight the COVID-19 menace. With respect to this, CSIR-CMERI is developing a motorized bellow-based ventilator, which has inbuilt intelligence to assist a patient who is trying to breathe or supply a prescribed volume at a defined pressure to a passive patient.
\end{abstract}

Keywords Acute respiratory distress syndrome (ARDS) $\cdot$ Tidal volume $\cdot$ Continous mandatory ventilation $\cdot$ Intermittent mandatory ventilation - PEEP · Passive and active patients - Invasive and non-invasive

\section{Introduction}

SARS-CoV-2, the virus that causes COVID-19, enters a healthy cell and uses the cell to make new viral parts. It multiplies, and the new viruses infect nearby cells. The new coronavirus can infect the upper or lower part of the respiratory tract and the infection may reach all the way down into the alveoli. The body immune system fights back as the infection travels down the respiratory tract. The lungs and airways swell and become inflamed. In some critical COVID-19 situations, the infection can damage the walls and linings of the air sacs in the lungs. As the body tries to fight it, the lungs become more inflamed and fill with fluid. This can make it harder for lungs to swap oxygen and carbon dioxide. One might have severe pneumonia or acute respiratory distress syndrome (ARDS). In the most critical cases, the lungs need help from a machine called a ventilator (https ://www.webmd.com/lung/what-does-covid-do-to-your-lungs).

The exponential growth of the patient suffering from COVID-19 has led to the rise in demand for the ventilators. One may estimate the requirement of 13.8 lacs ventilators based on the $10 \%$ rule. As per this estimation, India has a population of 138 crore and $10 \%$ of the population may be affected, if not controlled appropriately. Out of these, $10 \%$ people, 1.38 crore may have weak immune system and require hospitalization. Out of these, 10\% people, 13.8 lakh may experience ARDS and

H. Hirani

harishhirani6982@gmail.com

1 CSIR-Central Mechanical Research Institute, Durgapur, WB, India require ventilator. The ventilator should support both partially active and passive patients. This means the ventilators should have enough intelligence to sense breathing efforts of a partially active patient and assist the patient. Otherwise, it delivers the prescribed air volume (prescribed by doctor/nursing-staff).

\section{Recent Trends}

Many companies, research institute, engineers and innovators have pitched in for the development of ventilators using the Bag-Valve-Mask (BVM). Many innovative ideas are being used for the compression of the BVM to supply a fixed volume of air at a defined rate. However, the function of the ventilator is not just to supply air to the lungs, there are many criticalities involved while doing so. If a fixed volume of air is forced into the patient's lungs without understanding the patient's lung compliance, it may lead to a condition known as "Pulmonary Barotrauma" (Tobin 2013). This condition is nothing but damage to the lung from rapid or excessive airway pressure. The ventilator must have pressure-releasing mechanism to discharge excess pressure when limit on peak inspiratory pressure (PIP) is reached, thus protecting the lungs from severe damage.

\section{Mechanical Ventilation Pre-requisites}

A mechanical ventilator must have a continuous mandatory ventilation (CMV) mode, which must be one of following (https://assets.publishing.service.gov.uk/government/uploa ds/system/uploads/attachment_data/file/879382/RMVS0 01_v4.pdf): 
i. Pressure-regulated volume control (PRVC)

ii. Pressure-controlled ventilation (PCV)

iii. Minimally, a volume-controlled ventilation (VCV)

For a VCV, which is adopted in the present research, a tidal volume and respiratory rate are set. The tidal volume is delivered during the inspiratory period with following pressurelimiting controls:

- A micro-controller along with pressure sensors, which continuously monitors the change in inspiratory airway pressure, alarms when a set PIP limit $\left(15-70 \mathrm{cmH}_{2} \mathrm{O}\right.$ in increments of $5 \mathrm{cmH}_{2} \mathrm{O}$ ) is reached and reduces pressure thereafter automatically. In addition, there should be a mechanical fail-safe valve, which opens at $80 \mathrm{cmH}_{2} \mathrm{O}$ if there is electrical/electronic failure. Whenever any line in a breathing circuit is choked or disconnected, alarm turns on and an error message must be displayed on the screen. In addition, various waveforms related to patient-ventilator asynchrony must be displayed on the screen to understand the patient-ventilator asynchrony. Based on the results, desired changes in the setting may be implemented either by trained nursing staff or automatically.

- PEEP: ARDS led to the collapsing of the alveoli thereby reducing the functional residual capacity (FRC) of the lungs. The FRC acts as a buffer by preventing rapid changes in alveolar gas tensions from inspired air (Hess and Kacmarek 2014). A PEEP of 5-20 $\mathrm{cmH}_{2} \mathrm{O}$ adjustable in $5 \mathrm{cmH}_{2} \mathrm{O}$ increments needs to be maintained after the expiration.
- Inspiratory:expiratory ratio (I:E): the proportion of each breathing cycle that is spent "breathing in" compared to "breathing out" should preferably in an adjustable range of 1:1-1:3.

- Respiratory rate: the number of breathing cycles every minute, provided by ventilator, should be in a range of 10-30 breaths per minute in increments of 2 .

- Tidal volume setting: the volume of gas, flowing from ventilator into the lungs during one inspiratory cycle, should be in the range of 350-450 $\mathrm{ml}$ in steps of $50 \mathrm{ml}$.

\section{Basic Ventilator Developed by CSIR-CMERI}

The first prototype developed at CSIR-CMERI has used a Bag-Valve-Mask (BVM) to generate the compressed air required in a ventilator. However, during experiments, the following limitations were observed:

- Requirement of various sizes of BVM bags for different age group patients.

- Inconsistent flow from the BVM during compression at high respiratory rates. This may be due to the ellipsoidal shape of the BVM where it is easier for the air to get compressed rather than flowing out of it.

Due to these shortcomings, intermittent positive pressure ventilation (IPPV) system was designed using a cylindrical bellow (Fig. 1) to provide support to the patient in either invasive or non-invasive way, which is controlled and monitored with the help of a control system, sensors (flow and pressure),

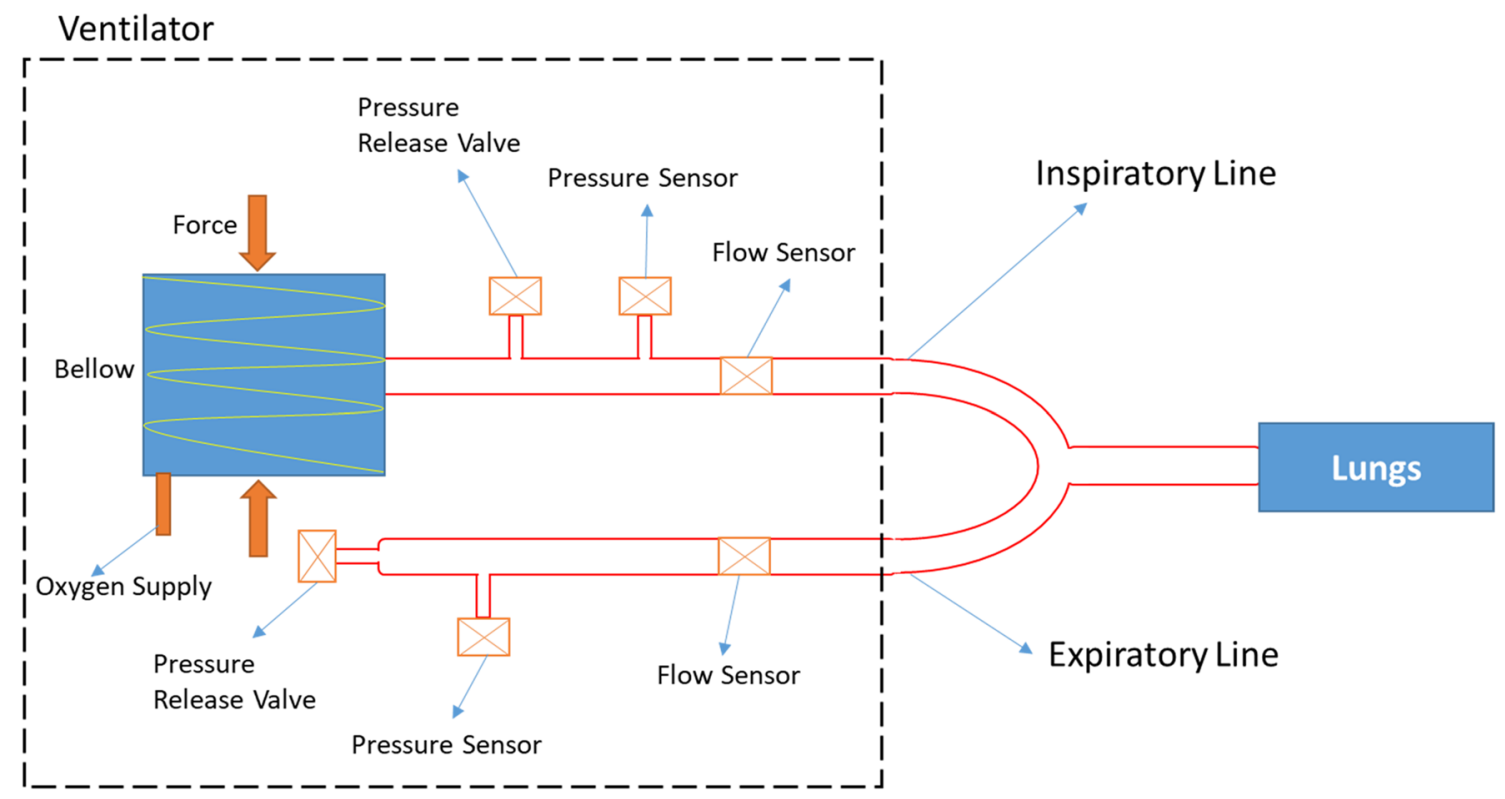

Fig. 1 Schematic layout diagram 
display and alarm. The device delivers air to the lungs at breathing rate and tidal volume as per the preset values. The device is run with a volume assist-control mode; hence, it can be used for both partially active and passive patients. Power is typically supplied from a power line or a UPS. This ventilator may be used for long-term respiratory support in extended care facilities, in home or even in emergency care.

\section{Principles of Operation}

In the developed prototype (Fig. 2), the bellow is compressed with the help of two arms, which are fixed on the rack. The rack is actuated with the help of a pinion, which is driven, with the help of a stepper motor. The required volume needed by the patient is controlled by changing the displacement of the two arms. An oxygen cylinder can also be attached to the bellow with the help of a pipe so that the required amount of fraction of inspired oxygen $\left(\mathrm{FiO}_{2}\right)$ can be controlled. The prototype has two lines (inspiratory line and expiratory line) as can be seen in schematic (Fig. 1). There are two pressure release valves in the system, one at expiratory line, which helps in maintaining the PEEP and another in inspiratory line, which helps in controlling the PIP. During the inspiration cycle when the preset volume is delivered by bellow to the lungs, it may happen that the peak airway pressure may rise due to the non-compliant lungs of the patient for the set volume. During these scenarios, control logic is set such that the excessive pressure will be released through the pressure release valve thus protecting the lungs from serious damages. The release in pressure leads to reduction in the volume getting into the lungs as compared to the set value, which is also being recorded, through the flow sensor. During the expiratory cycle, the pressure release valve releases pressure until the set value of PEEP is reached thus maintaining a positive pressure on the lungs. The pressure triggering mechanism in the ventilator also facilitates assistive breathing for partially active patient. The prototype comes with an alarm which is triggered when the pressure limit crosses or when the mechanism stops due to unforeseen circumstances.

\section{Specification}

Dimension: $300 \mathrm{~mm} \times 280 \mathrm{~mm} \times 330 \mathrm{~mm}$.

Weight: $\sim 5 \mathrm{~kg}$.

Tidal volume: $350-450 \mathrm{ml}$ (adjustable in steps of $50 \mathrm{ml})$.

Breathe rate: 10-30 BPM (adjustable).

I:E ratio: 1:1, 1:2, 1:3 (selectable).

Ventilator control mode: VCV with assist control (AC).

\section{Control System and Operations}

Arduino-based micro-controller is being used to actuate the arms of the mechanical ventilator based on the inputs provided by the user. The micro-controller is constantly being fed with the data from the sensors and thus helping in precise control of various parameter as well as actuation of the system when required. The salient features of the developed prototype are

- Breath cycle detection of a partially active patient through pressure sensing.

Fig. 2 Developed prototype

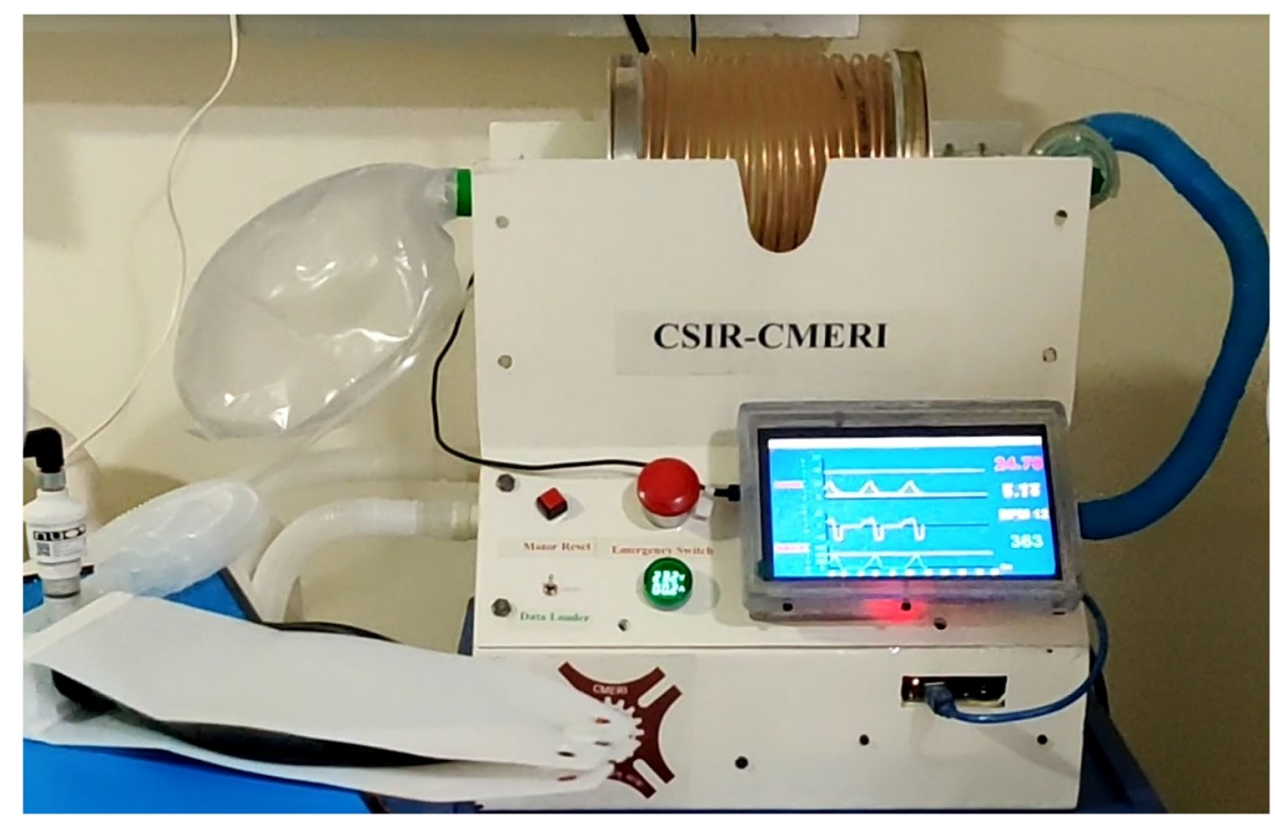


- Post-detection, supply of required quantity of air under volume assist/control mode during inhalation while monitoring that the PIP should not go beyond $40 \mathrm{~cm}$ of $\mathrm{H}_{2} \mathrm{O}$. Maintain a PEEP of $5 \mathrm{~cm}$ of $\mathrm{H}_{2} \mathrm{O}$ during the exhalation.

- The GUI has input fields for setting different parameters on the left side and the instantaneous measured values are displayed on the right side (Fig. 3).

- The system provides real-time display of lung pressure, flow and tidal volume delivered to the lung (Fig. 4).

- Assist-control mode operation: to identify whether patient is trying to breathe or not and then either assist or else supply the preset volume (Fig. 5). Please note the sudden dip in the PEEP (pressure vs time) due to the initiation of breath by patient.

\section{Testing}

The mechanical ventilation systems are classified as lifecritical systems, and every precaution must be taken to ensure that they are highly reliable, including their power supply. Therefore, the developed ventilator is to be tested as per "ISO 80601-2-12: 2020-02: Medical Electrical Equipment-Part 2-12: Particular Requirements for the Safety of Lung Ventilators-Critical Care Ventilators" to prove its basic performance and usability. However, it is acceptable that full demonstration of compliance to ISO 80601-2-12:2020 is unrealistic in the time frame required for development (https://assets.publishing.service.gov.uk/
Fig. 3 GUI of the developed software

Fig. 4 Real-time display of pressure, flow and tidal volume
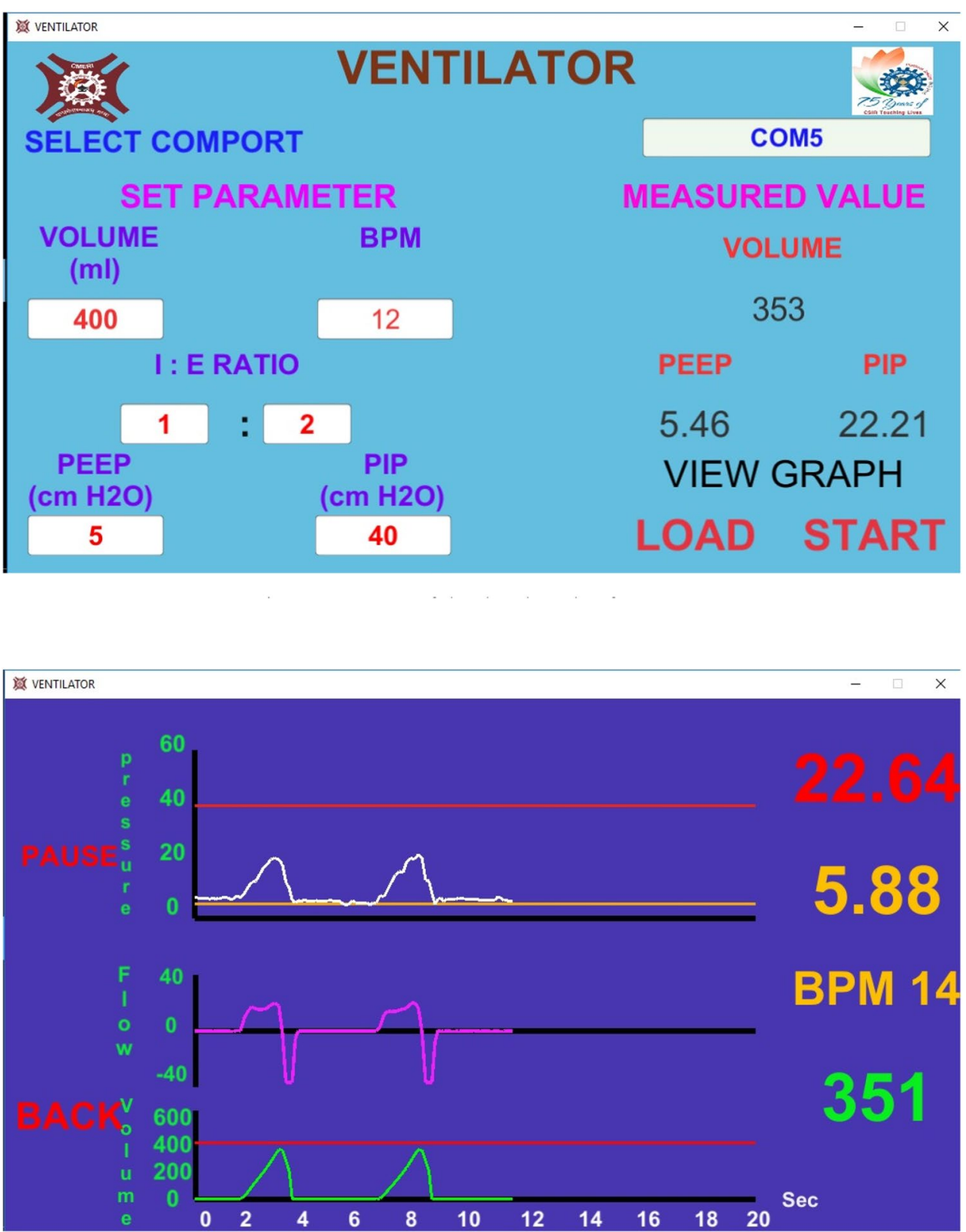
Fig. 5 Sensing of patient breathing effort, simulated by stretching the test lung

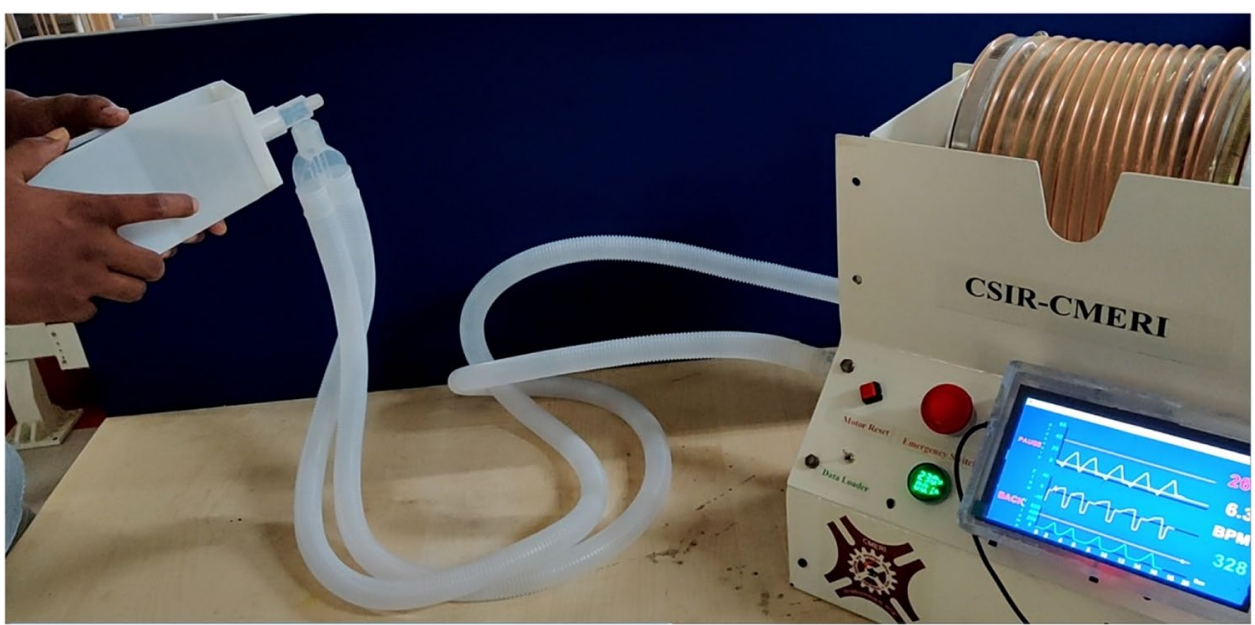

government/uploads/system/uploads/attachment_data/ file/879382/RMVS001_v4.pdf). Nevertheless, compliance with the essential safety standards must be demonstrated for patient safety. For this purpose, a suitable testing rig is to be developed where the basic performance of the developed ventilator may be demonstrated. In addition to this, various other tests for compliance are also needed as per different IEC and ISO standards (Fig. 6).

The developed prototype has been tested extensively in a hospital setup under the observations of three Anesthesiologists of Health World Hospitals, Durgapur.

\section{Conclusions}

During the current COVID-19 pandemic, the requirement for ventilators has multiplied many folds, forcing various industries, innovators and engineers to innovate ideas which can be mass produced to meet the market demand. However, it would be blatantly incorrect to assume a ventilator for just providing a set volume of air at a set breath rate. In COVID19, patients may suffer from a condition like ARDS, which calls for a ventilator with at least one mode, i.e., assist-control mode with a mandatory option of patient triggering and maintaining of PEEP at the end of expiratory cycle. In addition, the system should be able to handle a slightly higher peak inspiratory pressure (PIP), up to $70 \mathrm{~cm}$ of $\mathrm{H}_{2} \mathrm{O}$ due to ARDS (Lei 2017). None of the BVM-based ventilators that are available today can integrate this mode into their system. Using these ventilators on the patient will do more harm than good. Moreover, the software is also likely to be a high-risk device that will almost certainly have the capability to cause serious injury or death because of the accelerated development cycle. Ideally, the software code should be written by following the "IEC 62304: 2015: Medical Device Software-Software Life Cycle Processes" and it should be developed in a facility that has experience of developing software using different applicable standards (https://asset
Fig. 6 Ventilator trial is going on at Health World Hospitals, Durgapur

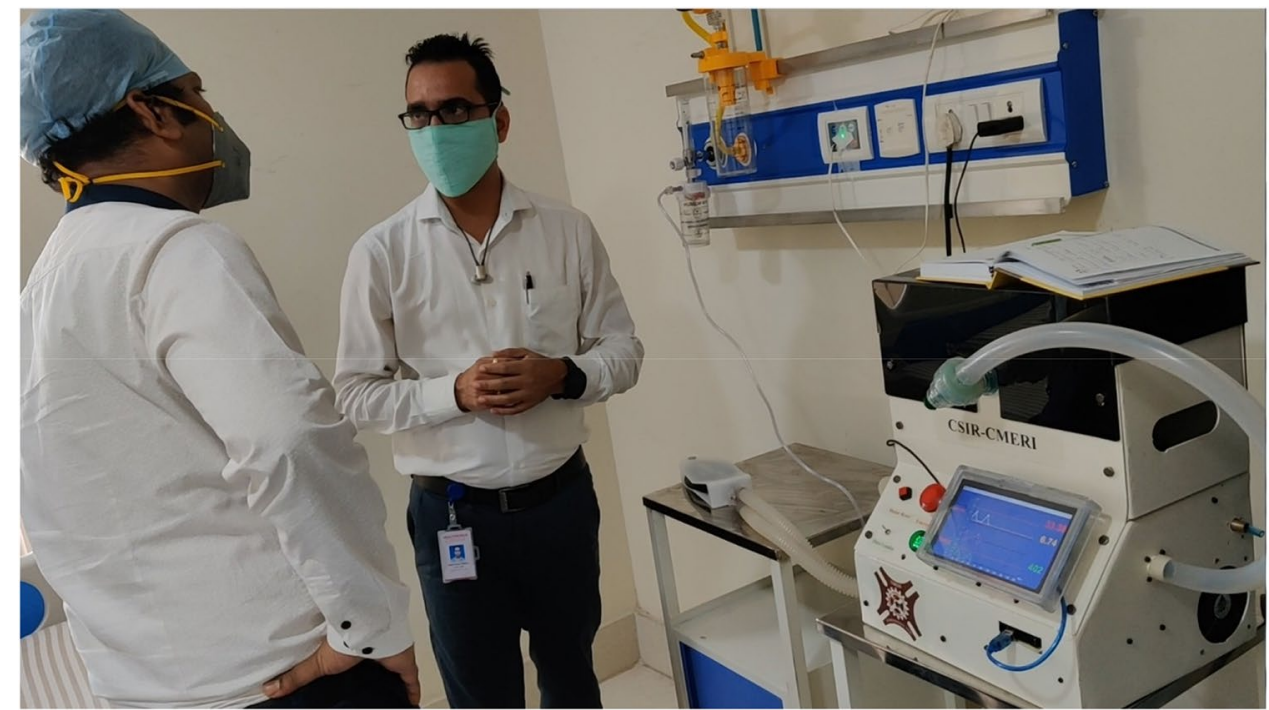


s.publishing.service.gov.uk/government/uploads/system/ uploads/attachment_data/file/879382/RMVS001_v4.pdf). This paper is an attempt for the development of easily massproduced bellow-based ventilator having the bare minimum specification, which may be used in the present pandemic scenario. However, rigorous testing of the developed ventilators has to be done as per ISO 80601-2-12:2020; system shortcomings need to be understood and redesign needs to be carried out.

\section{Future work}

The development work is continuing for another ventilator, which supports both pressure- and volume-based ventilation and this will be comparable to the ICU ventilators. Further research is going on to integrate an Oxygen Enrichment Unit with the ventilator so that it can be used in any locations even outside a hospital if such a need arises.

Acknowledgements The author acknowledges the untiring efforts of the scientists of CSIR-CMERI during the lockdown period to develop innovative and cost-effective products that can be of great help for the common people to fight the COVID-19 pandemic. Special mention goes to Dr. Anupam Sinha, Dr. Sudip Samanta, Sanjay Hansdah and Kalyan Chatterjee for their involvement in developing the mechanical ventilator. In addition, the author also acknowledges the inputs provided by Dr. Arindam Hazra, Dr. Brojen Choudhury and Dr. Ramprasad Gorai of Health World Hospitals, Durgapur, during the course of development.

\section{References}

Hess DR, Kacmarek RM (2014) Essentials of Mechanical Ventilation, 3rd edn. McGraw-Hill Education, New York

Lei Y (2017) Medical ventilator system basics-a clinical guide. Oxford University Press, Oxford

Rapidly manufactured ventilator system. Medicines and Healthcare products Regulatory Agency, UK. https://assets.publishing.servi ce.gov.uk/government/uploads/system/uploads/attachment_data/ file/879382/RMVS001_v4.pdf

Tobin MJ (2013) Principles and practice of mechanical ventilation, 3rd edn. McGraw-Hill, New York

What does COVID-19 do to your lungs? A WebMD publication. https ://www.webmd.com/lung/what-does-covid-do-to-your-lungs

Publisher's Note Springer Nature remains neutral with regard to jurisdictional claims in published maps and institutional affiliations. 